\title{
Muscle-specific E3 ubiquitin ligases are involved in muscle atrophy of cancer cachexia: An in vitro and in vivo study
}

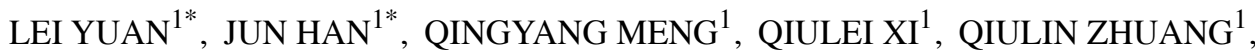 \\ YI JIANG $^{1}$, YUSONG HAN ${ }^{1}$, BO ZHANG ${ }^{1}$, JING FANG ${ }^{2}$ and GUOHAO WU ${ }^{1}$ \\ ${ }^{1}$ Department of General Surgery, Zhongshan Hospital, Fudan University; ${ }^{2}$ Key Laboratory of Food Safety Research, \\ Institute for Nutritional Sciences, Shanghai Institutes for Biological Sciences, \\ Chinese Academy of Sciences, Shanghai, P.R. China
}

Received December 8, 2014; Accepted February 9, 2015

DOI: 10.3892/or.2015.3845

\begin{abstract}
Muscle atrophy F-Box (MAFbx)/atrogin-1 and muscle ring-finger-1 (MuRF-1) have been identified as two muscle-specific E3 ubiquitin ligases that are highly expressed in skeletal muscle during muscle atrophy. However, the role of muscle-specific E3 ubiquitin ligases during the process of muscle atrophy of cancer cachexia remains largely unknown. In the present study, we analyzed the expression of atrogin-1 and MuRF-1 in the skeletal muscle of patients with malignant and benign disease. The possible mechanisms were studied both in a colon 26-induced cancer cachexia mouse model and in tumor necrosis factor- $\alpha(\mathrm{TNF}-\alpha)$ induced atrophy $\mathrm{C} 2 \mathrm{C} 12$ cells. Our results demonstrated that atrogin- 1 and MuRF-1 tended to be increased in the skeletal muscle of patients with malignant disease even before weight loss. Non-tumor body weights and gastrocnemius weights were significantly decreased while expression levels of ubiquitin proteasome pathway associated genes (atrogin-1, MuRF-1, ubiquitin and E2-14K) were upregulated in cancer cachexia mice. Significant myotube atrophy with atrogin-1 overexpression was observed in the $\mathrm{C} 2 \mathrm{C} 12$ cells treated with TNF- $\alpha$. Meanwhile, knockdown of atrogin-1 by small interfering RNA (siRNA) protected C2C12 cells from the adverse effect of TNF- $\alpha$. In conclusion, muscle-specific E3 ubiquitin ligases were upregulated during cancer cachexia, and atrogin-1 may be a potential molecular target for treating muscle atrophy induced by cancer cachexia.
\end{abstract}

Correspondence to: Professor Guohao Wu, Department of General Surgery, Zhongshan Hospital, Fudan University, 180 Fenglin Road, Shanghai 200032, P.R. China

E-mail:prowugh@163.com

${ }^{*}$ Contributed equally

Key words: cancer cachexia, muscle atrophy, atrogin-1, MuRF-1, tumor necrosis factor- $\alpha$, small interfering RNA

\section{Introduction}

Cancer cachexia, a condition of advanced protein calorie malnutrition, is characterized by loss of skeletal muscle protein, depletion of lipid storage, anorexia, poor performance and ultimately death (1-3). Cancer cachexia occurs in $\sim 80 \%$ of cancer patients and is the primary cause of death for $22-30 \%$ of all cancer patients $(3,4)$. However, cancer cachexia is difficult to diagnose until patients have lost more than 5-7\% of their body weight $(5,6)$. Furthermore, it was reported that $80 \%$ of patients with upper gastrointestinal cancers and $60 \%$ of patients with lung cancer had already experienced substantial weight loss at the moment of diagnosis (7). Therefore, it is essential to illuminate the early mechanisms in the development of cancer cachexia.

The pathogenesis of cancer cachexia including alterations in energy and substrate metabolisms is multifunctional. Skeletal muscle protein depletion (i.e. muscle atrophy) due to hyper-catabolism is the most common characteristic of cancer cachexia. Cancer cachexia is a complex process that occurs as a consequence of a variety of stressors including neural inactivity, mechanical unloading, inflammation, metabolic stress and elevated glucocorticoids $(1,8,9)$. Several proteolytic systems, such as the lysosome, the calcium-dependent systems and the ubiquitin-dependent systems are involved in degrading skeletal muscle (8). However, it was reported that ubiquitin dependent proteolysis is the main mechanism for the enhanced muscle protein degradation in cancer cachexia (10). In addition, cytokines such as tumor necrosis factor- $\alpha$ (TNF- $\alpha$ ), interleukin-1 (IL-1), interleukin-6 (IL-6) and interferon- $\gamma$ (IFN- $\gamma$ ) have been proposed as mediators for the cancer cachectic process $(3,11,12)$.

The ubiquitin-dependent proteasome pathway includes a series of reactions involving three classes of proteins: ubiquitin-activating enzymes (E1), ubiquitin-conjugating enzymes (E2), and ubiquitin protein ligases (E3) (13). Muscle atrophy F-Box (MAFbx)/atrogin-1 and muscle ring-finger-1 (MuRF-1), identified over 10 years ago, are two muscle-specific E3 ubiquitin ligases that are increased in skeletal muscle under atrophy-inducing conditions such as humans under voluntary bed rest (14) or with spinal cord injury (15), amyotrophic lateral sclerosis (16), chronic obstructive pulmonary disease 
(COPD) (17) and in animal models of aging (18), diabetes (19), starvation (20), making them excellent markers for muscle atrophy. However, little is known concerning the role of muscle-specific E3 ubiquitin ligase genes during the process of cancer cachexia.

The goal of the present study was to elucidate the role of muscle-specific E3 ubiquitin ligases during cancer cachexia in vitro and in vivo. Firstly, we determined the expression of muscle-specific E3 ubiquitin ligases both in patients with malignant and benign disease. Furthermore, in a mouse model of cancer cachexia, we analyzed the expression of musclespecific E3 ubiquitin ligases and other ubiquitin proteasome pathway-associated genes. Finally, we illustrated the protective effect of atrogin-1 small interfering RNA (siRNA) in an atrophy model of muscle cells induced by TNF- $\alpha$. Our results showed that muscle-specific E3 ubiquitin ligases were upregulated both in humans with malignant disease and mice with cancer cachexia. Atrogin-1 gene silencing protected myotubes from atrophy induced by TNF- $\alpha$. We believe that such a comprehensive analysis of muscle-specific E3 during cancer cachexia in clinical patients to an animal model and muscle cells illuminated the importance of muscle-specific E3 during cancer cachexia and may provide novel targets for drug discovery in the intervention of the progression of cancer cachexia.

\section{Materials and methods}

Patients and muscle samples. Twenty-one patients with malignant disease admitted to Zhongshan Hospital of Fudan University between August 2011 and December 2013 were enrolled in the present study. Diagnoses of malignant disease were confirmed by postoperative pathological examinations. Twenty-three patients undergoing surgery for benign abdominal diseases served as the control group. Exclusion criteria were patients with acute or chronic renal and liver failure, acute and chronic hepatitis, diabetes, metabolic acidosis, sepsis, AIDS, inflammatory bowel disease, autoimmune disorders, chronic heart failure, hyperthyroidism and chronic obstructive pulmonary disease. Written informed consent was obtained from all patients, and permission for the present study was obtained from the Ethics Committee of the Zhongshan Hospital, Fudan University.

The nutritional assessments of patients before surgery included height, actual body weight, body mass index (BMI), total lymphocyte count, serum albumin and prealbumin. A biopsy specimen was obtained from the rectus abdominis muscles according to the methods described by Bossola et al (21). The biopsy specimen was immediately frozen in liquid nitrogen and then stored at $-70^{\circ} \mathrm{C}$ until further analysis. No complications occurred during the biopsy procedure.

Animals and treatments. BALB/c male mice (6-8 week of age) weighing 16-20 g were purchased from the Shanghai Laboratory Animal Center, Chinese Academy of Sciences. Mice were housed at $22 \pm 1^{\circ} \mathrm{C}$, with a 12 -h light/dark cycle, free access to water and conventional diet. Mice were acclimated to the environment for one week before the study started. All animal manipulations were carried out according to the guidelines of regulations for the use of experimental animals of the Chinese Academy of Science. All efforts were made to minimize animal suffering and to use only the number of animals necessary to produce reliable scientific data.

Colon 26/clone 20 cells, which have been reported to induce severe cachexia in BALB/c mice by subcutaneous inoculation, were purchased from the Shanghai Institute of Pharmaceutical Industry. The cells were cultured in RPMI-1640 supplemented with $5 \%$ fetal bovine serum and $1 \%$ penicillin-streptomycin at $37^{\circ} \mathrm{C}$ in $5 \% \mathrm{CO}_{2}$. On study day $0,1.0 \times 10^{6}$ cells suspended in $100 \mathrm{ml}$ phosphate-buffered saline (PBS) were injected subcutaneously into the armpits of the mice in the tumor group. An equal volume of PBS without tumor cells was injected into the control group mice. The body weight of each mouse was recorded every two days after tumor cell inoculation. On day 16 , the mice were sacrificed via cervical dislocation. Non-tumor body weights and gastrocnemius muscle weights were recorded. Muscle specimens were frozen at $-70^{\circ} \mathrm{C}$ for further analysis.

Cell lines and treatment in vitro. Mouse skeletal muscle $\mathrm{C} 2 \mathrm{C} 12$ cells were propagated and maintained in Dulbecco's modified Eagle's medium (DMEM) supplemented with antibiotics and $10 \%$ fetal bovine serum at $37^{\circ} \mathrm{C}$ in the presence of $5 \% \mathrm{CO}_{2}$. To induce differentiation, $\mathrm{C} 2 \mathrm{C} 12$ cells were propagated in DMEM supplemented with antibiotics and $2 \%$ horse serum for the indicated period. After 3 days of differentiation, according to a previous report (22), $\mathrm{C} 2 \mathrm{C} 12$ cells were treated with $10 \mathrm{ng} / \mathrm{ml}$ recombinant mouse TNF- $\alpha$ (R\&D Systems, USA) for $96 \mathrm{~h}$ to extract proteins, and $\mathrm{C} 2 \mathrm{C} 12$ cells were treated with $6 \mathrm{ng} / \mathrm{ml}$ recombinant mouse TNF- $\alpha$ for $2 \mathrm{~h}$ to extract RNAs.

Construction and infection of the lentivirus containing siRNA targeting the human atrogin-1 gene. Five pairs of siRNAs targeting the human atrogin-1 gene (Table I) were designed using Ambion siRNA Target Finder and were synthesized by Invitrogen Biotechnology Co., Ltd. (Shanghai, China). In addition, negative control siRNA was also synthesized for monitoring the influence of the exogenous genes. These siRNAs were cloned into vector pBS-hU6-I and then into vector FG12. The length and accuracy of the sequences were confirmed by DNA sequencing. The recombinant FG12 vectors were cotransfected with pRSVREV, pMDLg/pRRE and pHCMV-G into 293T cells to package lentivirus particles, with which $\mathrm{C} 2 \mathrm{C} 12$ cells were infected. The infected $\mathrm{C} 2 \mathrm{C} 12$ cells were cultured and differentiated to form myotubes before TNF- $\alpha$ was added to induce atrophy. Atrogin-1 mRNA and protein were identified by real-time PCR (RT-qPCR) and western blotting, respectively. Myotube morphology was observed and photographed directly in the culture plate without fixation.

Western blot analysis. Total proteins were extracted from cells or muscle tissues and quantified by the BCA method. Western blotting was performed to determine the protein expression of atrogin-1. The protocol used for western blotting was previously described (23). Briefly, $50 \mathrm{mg}$ total proteins was separated by SDS-PAGE, transferred to PVDF membranes, and analyzed by western blotting with a goat polyclonal anti-human atrogin-1 antibody (Santa Cruz Biotechnology, USA). An antibody against $\alpha$-tubulin (Santa Cruz Biotechnology) served as an endogenous control. Finally, the membrane was visualized by an imaging system in the dark to quantify the protein level. 
Table I. Primers of atrogin-1 siRNAs.

\begin{tabular}{|c|c|c|}
\hline No. & $\begin{array}{l}\text { Sense } \\
\text { Antisense }\end{array}$ & Sequences \\
\hline \multirow[t]{2}{*}{1} & Sense & 5'ACCGGAGGTATACAGTAAGGAGTTCAAGAGACTCCTTACTGTATACCTCCTTTTTGGATCCC3' \\
\hline & Antisense & 5'TCGAGGGATCCAAAAAGGAGGTATACAGTAAGGAGTCTCTTGAACTCCTTACTGTATACCTC3' \\
\hline \multirow[t]{2}{*}{2} & Sense & 5'ACCGCTTTCAACAGACTGGACTTTCAAGAGAAGTCCAGTCTGTTGAAAGCTTTTTGGATCCC3' \\
\hline & Antisense & 5'TCGAGGGATCCAAAAAGCTTTCAACAGACTGGACTTCTCTTGAAAGTCCAGTCTGTTGAAAG3 \\
\hline \multirow[t]{2}{*}{3} & Sense & 5'ACCGGCTGTTGGAGCTGATAGCTTCAAGAGAGCTATCAGCTCCAACAGCCTTTTTGGATCCC3' \\
\hline & Antisense & 5'TCGAGGGATCCAAAAAGTCTGTGCTGGTGGGCAACTCTCTTGAAGTTGCCCACCAGCACAGA3 \\
\hline \multirow[t]{2}{*}{4} & Sense & 5'ACCGTCTGTGCTGGTGGGCAACTTCAAGAGAGTTGCCCACCAGCACAGACTTTTTGGATCCC3' \\
\hline & Antisense & 5'TCGAGGGATCCAAAAAGTCTGTGCTGGTGGGCAACTCTCTTGAAGTTGCCCACCAGCACAGA3 \\
\hline \multirow[t]{2}{*}{5} & Sense & 5'ACCGCGTTTGATCTTGTCTGACTTCAAGAGAGTCAGACAAGATCAAACGCTTTTTGGATCCC3' \\
\hline & Antisense & 5'TCGAGGGATCCAAAAAGCGTTTGATCTTGTCTGACTCTCTTGAAGTCAGACAAGATCAAACG3' \\
\hline \multirow[t]{2}{*}{ Ctrl } & Sense & 5'ACCGATGTTGTCAACGACTAGTTTCAAGAGAACTAGTCGTTGACAACATCTTTTTGGATCCC3' \\
\hline & Antisense & 5'TCGAGGGATCCAAAAAGATGTTGTCAACGACTAGTTCTCTTGAAACTAGTCGTTGACAACAT3' \\
\hline
\end{tabular}

siRNAs, small interfering RNAs; Ctrl, control.

Table II. Primer sequences of the target genes and $\beta$-actin.

\begin{tabular}{llll}
\hline Species & Genes & \multicolumn{1}{c}{ Forward primers } & Reverse primers \\
\hline Human & Atrogin-1 & GACTTCTCAACTGCCATTC & TCGTCTCCATCCGATACAC \\
Human & MuRF-1 & GCTGAGCCAGAAGTTTGA & CAGGGCGTCTGCTATGTG \\
Human & $\beta$-actin & GATCATTGCTCCTCCTGAGC & ACTCCTGCTTGCTGATCCAC \\
Mouse & Atrogin-1 & CTGGATTGGAAGAAGATGTA & CTTGAGGGGAAAGTGAGACG \\
Mouse & MuRF-1 & CCTACTTGCTCCTTGTGC & TCCTGCTCCTGCGTGAT \\
Mouse & Ubiquitin & GACAGGCAAGACCATCACC & CACCCAAGAACAAGCACAA \\
Mouse & E2-14K & TGGACCAGAAGGGACACC & TGGCTGGACTGTTTGGATT \\
Mouse & $\beta$-actin & AGGTGTGCACCTTTTATTGGTCTCAA & TCCCTCTGGTTTGGAAGTATGT \\
\hline
\end{tabular}

MuRF-1, muscle ring-finger-1.

RNA extraction and RT-qPCR analysis. Total RNAs were isolated from the cells or tissues with TRIzol (Invitrogen, Carlsbad, CA, USA). RNA integrity was detected by an agarose gel method, and the RNA concentration was quantified by measuring A260 and A280 absorbance in a NanoDrop spectrophotometer (Thermo Scientific, USA). cDNA synthesis was carried out with $2 \mu \mathrm{g}$ of total RNAs by reverse transcription (RT) (Promega, Madison, WI, USA). RT-qPCR was performed using 7900HT Fast Real-Time PCR System (Applied Biosystems, Inc., Foster City, CA, USA). Real-time Master Mix (Toyobo, Osaka, Japan) was used to detect and quantify the expression levels of the target genes. The PCR reaction consisted of an initial denaturation at $95^{\circ} \mathrm{C}$ for $10 \mathrm{~min}$ followed by 40 cycles of $30 \mathrm{sec}$ at $95^{\circ} \mathrm{C}, 10 \mathrm{sec}$ at $60^{\circ} \mathrm{C}$ and $30 \mathrm{sec}$ at $72^{\circ} \mathrm{C}$, and finally $10 \mathrm{~min}$ at $72^{\circ} \mathrm{C}$. Amplification of the target cDNA was normalized to $\beta$-actin expression. Relative levels of target mRNA expression were calculated using the $2^{-\Delta \Delta \mathrm{Ct}}$ method. The primers used are listed in Table II.
Immunohistochemical staining. Immunohistochemical staining (IHC) of the tissues was performed as previously described (23). Briefly, muscle tissues were fixed in $4 \%$ formalin for $24 \mathrm{~h}$ and paraffin-embedded using a conventional method. Sections $(3-\mu \mathrm{m})$ were dehydrated in xylene and graded alcohols. Antigen retrieval was performed with $0.01 \mathrm{M}$ citrate buffer at $\mathrm{pH} 6.0$ at $95^{\circ} \mathrm{C}$ for $20 \mathrm{~min}$. The slides were then incubated with atrogin- 1 antibody at $4^{\circ} \mathrm{C}$ overnight and then with the fluorescein isothiocyanate (FITC)-conjugated secondary antibodies for $60 \mathrm{~min}$ at room temperature in the dark.

Statistical analysis. Statistical analysis was performed using GraphPad Prism 5.0 software (GraphPad Software, USA). Continuous variables were compared using the Student's t-test and are expressed as the mean and standard deviation (SD). $\mathrm{P}<0.05$ was considered to indicate a statistically significant result. 

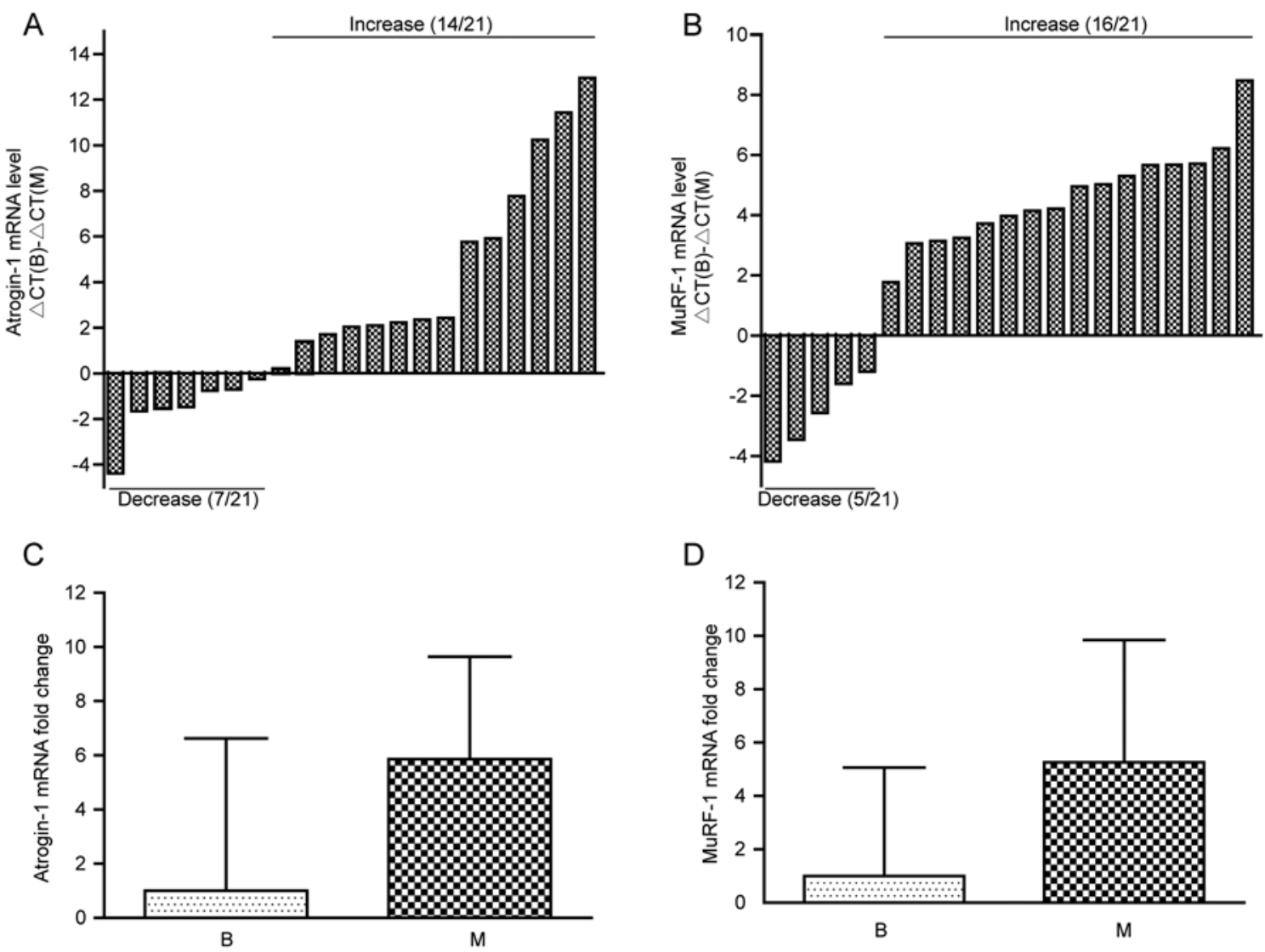

Figure 1. Atrogin-1 and MuRF-1 mRNA expression in rectus abdominis muscle of patients with malignant disease and benign disease. (A and B) Determination of atrogin-1 and MuRF-1 mRNA by real-time PCR (RT-qPCR) showed that atrogin-1 and MuRF-1 mRNA levels were increased in 66.7\% (14/21) and $76.2 \%(16 / 21)$ of the malignant disease patients compared to the benign disease patients, respectively. $\Delta \mathrm{Ct}(\mathrm{M})$ was calculated by subtracting the $\mathrm{Ct}$ value of $\beta$-actin from the $\mathrm{Ct}$ value of atrogin-1 (or MuRF-1) in each malignant disease patient. $\Delta \mathrm{Ct}$ (B) represents the mean $\Delta \mathrm{Ct}$ of all benign disease patients. (C and D) Determination of atrogin-1 and MuRF-1 mRNA by RT-qPCR showed that atrogin-1 and MuRF1 tended to be increased in the 16 malignant disease patients compared to the 23 benign disease patients without weight loss $(\mathrm{P}>0.05)$. M, malignant disease patients; $\mathrm{B}$, benign diseases patients. MuRF-1, muscle ring-finger-1.

\section{Results}

Atrogin-1 and MuRF1 mRNA are highly expressed in the patients with malignant disease. The clinical characteristics of the patients with malignant disease and benign disease are shown in Table III. Nutrition statuses such as BMI, total lymphocyte count, serum albumin and pre-albumin were significantly different in the two groups $(\mathrm{P}<0.05)$, while other characteristics showed no significant differences.

The mRNA levels of atrogin- 1 and MuRF-1 in the rectus abdominis muscles were analyzed by RT-qPCR. Considering the mean $\Delta \mathrm{Ct}$ of the benign disease group as control, we determined the $\Delta \mathrm{Ct}$ of atrogin-1 and MuRF-1 in the malignant disease group. As shown in Fig. 1A and B, atrogin-1 and MuRF-1 mRNA levels were increased in 66.7\% (14/21) and $76.2 \%(16 / 21)$ of the malignant disease patients, respectively. Univariate analysis indicated that the atrogin- 1 and MuRF-1 mRNA levels were significantly highly expressed in the malignant disease patients $(\mathrm{P}<0.05$, data not shown). In addition, to determine whether the expression of atrogin-1 and MuRF-1 mRNA increased before weight loss, we compared the mRNA levels of atrogin-1 and MuRF-1 in 16 malignant disease patients and 23 benign disease patients without weight loss. The results showed that both atrogin-1 and MuRF-1 mRNA
Table III. Characteristics of the included patients.

\begin{tabular}{lccc}
\hline & $\begin{array}{c}\text { Malignant } \\
\text { disease } \\
\text { group } \\
(\mathrm{n}=21)\end{array}$ & $\begin{array}{c}\text { Benign } \\
\text { disease } \\
\text { group } \\
(\mathrm{n}=23)\end{array}$ & P-value \\
\hline Mean age, years & $59.4 \pm 11.2$ & $53.8 \pm 10.9$ & $\mathrm{NS}$ \\
Gender (M:F) & $14: 7$ & $17: 6$ & $\mathrm{NS}$ \\
Weight, kg & $61.1 \pm 9.2$ & $63.4 \pm 10.4$ & $\mathrm{NS}$ \\
BMI & $20.5 \pm 2.9$ & $22.5 \pm 3.1$ & 0.03 \\
Weight loss, & $6 / 21$ & $0 / 23$ & $<0.001$ \\
n/total & & & \\
Total lymphocyte & & & \\
count, x109/1 & $1.5 \pm 0.7$ & $1.6 \pm 0.6$ & $\mathrm{NS}$ \\
Serum albumin, g/1 & $39.9 \pm 5.3$ & $44.2 \pm 4.3$ & 0.004 \\
Serum pre-albumin, g/1 & $0.21 \pm 0.06$ & $0.28 \pm 0.04$ & $<0.001$ \\
& & &
\end{tabular}

NS, not significant; M, male; F, female; BMI, body mass index.

levels tended to increase in the malignant disease patients but without statistical difference $(\mathrm{P}=0.099, \mathrm{P}=0.132$, respectively, Fig. 1C and D). 
A

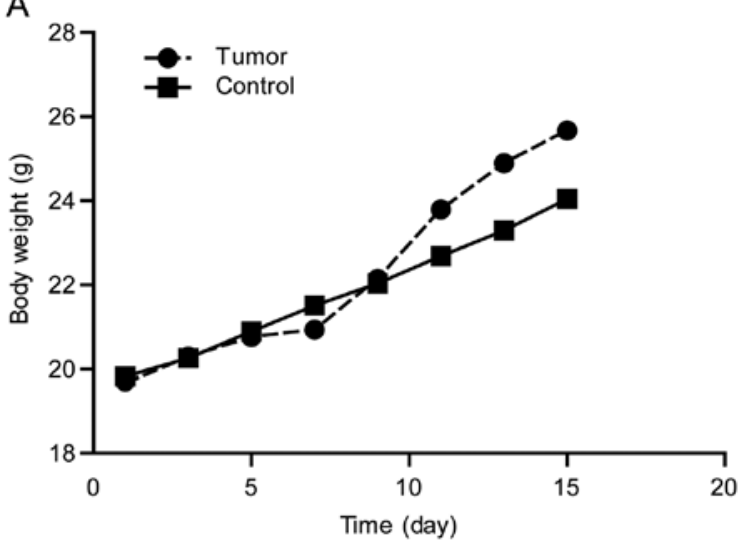

B

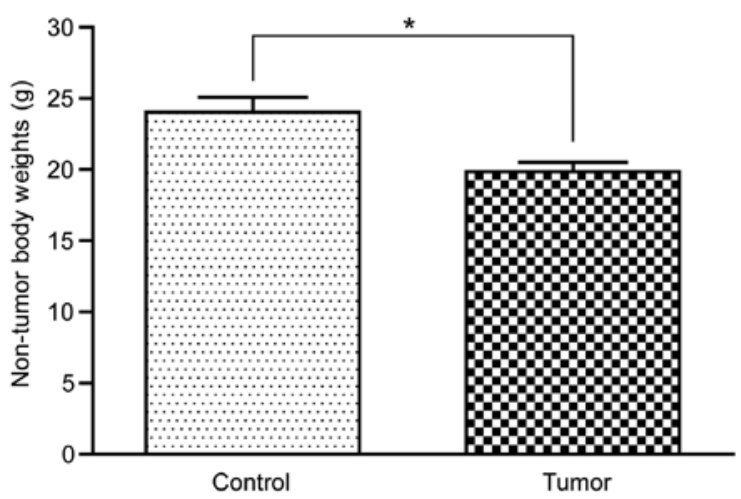

C

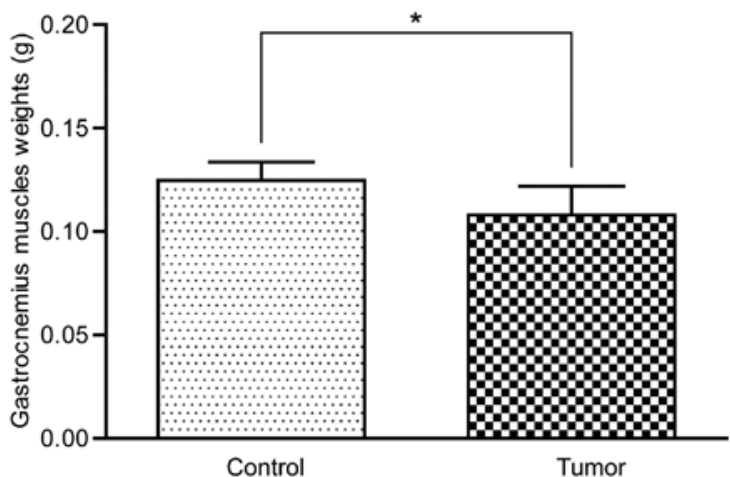

A

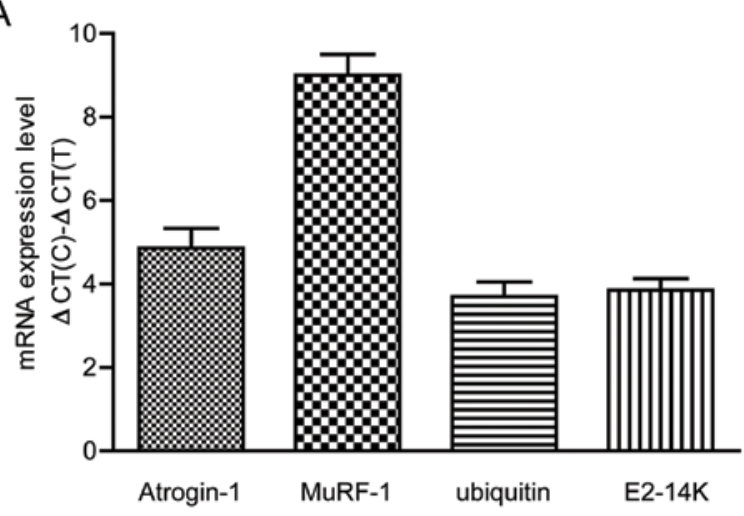

B

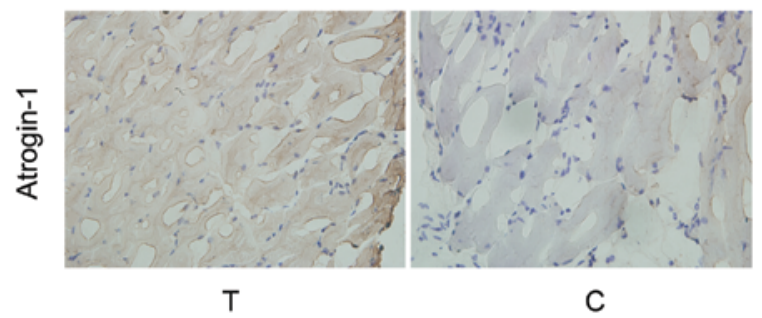

C

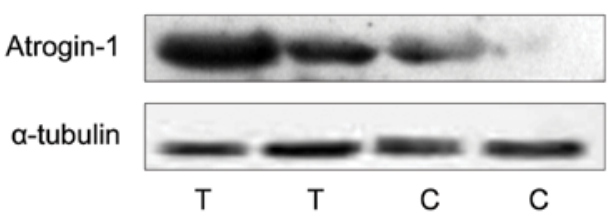

Figure 3. Expression of four ubiquitin proteasome pathway-associated genes (atrogin-1, MuRF-1, ubiquitin and E2-14K) in gastrocnemius muscles of mice. (A) Determination of atrogin-1, MuRF-1, ubiquitin and E2-14K mRNA levels by RT-qPCR showed that levels of all four genes were increased in the tumor-bearing mice compared to the non-tumor mice (control). $\Delta \mathrm{Ct}$ (C) represents the mean $\Delta \mathrm{Ct}$ of each mouse in the control group. $\Delta \mathrm{Ct}$ (T) represents the mean $\Delta \mathrm{Ct}$ of each tumor-bearing mouse. (B) Determination of atrogin-1 protein by immunohistochemical staining showed that atrogin-1 protein was overexpressed in the tumor-bearing mice (magnification, $\mathrm{x} 400$ ). (C) Determination of atrogin-1 protein by western blotting showed that atrogin-1 protein was overexpressed in the tumor-bearing mice. $\mathrm{T}$, tumorbearing mice; $\mathrm{C}$, non-tumor mice (control). MuRF-1, muscle ring-finger-1.

than the control group (Fig. 2B and C), indicating that cancer cachexia was effectively induced in this model.

Ubiquitin proteasome pathway-associated genes are highly expressed in the cachexia mice. We determined the expression of four ubiquitin proteasome pathway-associated genes (atrogin-1, MuRF-1, ubiquitin and E2-14K) in the muscles of colon-26-induced cachexia mice. Compared to the nontumor mice, atrogin-1, MuRF-1, ubiquitin and E2-14K mRNA levels were significantly increased in the cachexia mice $(\mathrm{P}<0.05$, Fig. $3 \mathrm{~A})$. Western blotting and IHC confirmed that the atrogin-1 protein was overexpressed in the cachexia mice (Fig. 3B and C).

Optimization of the lentiviral vector system for siRNAs targeting atrogin-1. The accuracy of the recombinant plasmids 


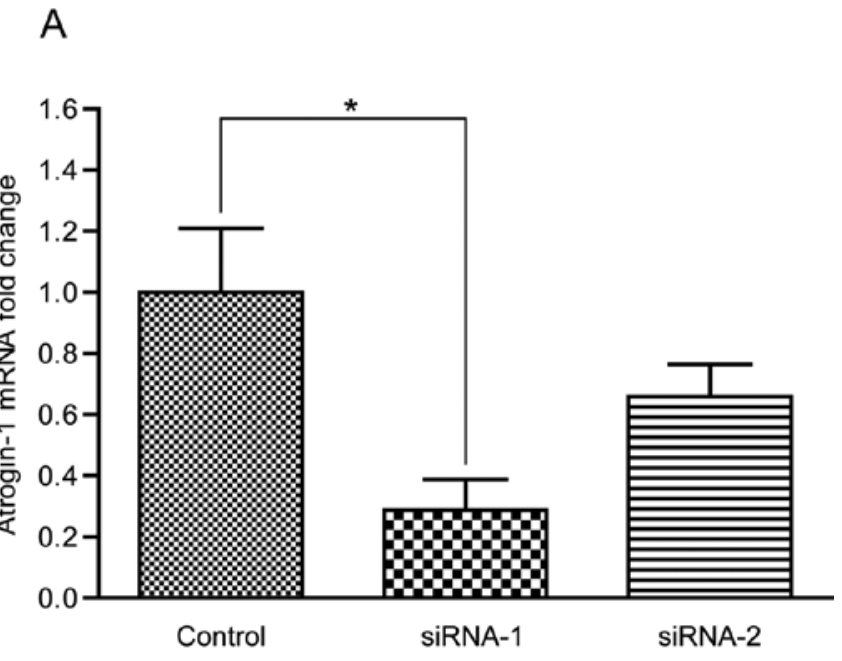

B

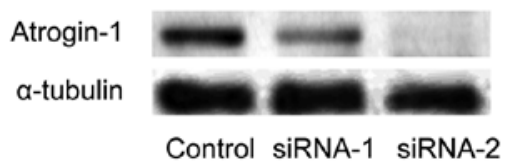

Figure 4. Atrogin-1 expression after transfection with atrogin-1 siRNA-1 and -2. (A) Determination of the atrogin-1 mRNA level by RT-qPCR showed that atrogin-1 siRNA-1 and -2 decreased atrogin-1 mRNA compared to the non-specific siRNA (control). (B) Determination of atrogin-1 protein by western blotting showed the downregulation of atrogin-1 protein after transfection with atrogin-1 siRNA-1 and -2 . "Significant difference $(\mathrm{P}<0.05)$ between the two groups.

was confirmed by DNA sequencing. Two correct recombinant plasmids PBSU6I-atrogin-1 siRNA-1 and -2 were chosen for further analysis, while three incorrect recombinant plasmids PBSU6I-atrogin-1 siRNA-3, -4 and -5 were discarded. After transfection with the recombinant lentivirus vector expressing atrogin-1 siRNA-1 and -2, atrogin-1 was downregulated in the $\mathrm{C} 2 \mathrm{C} 12$ cells. However, only atrogin-1 siRNA-1 was identified to be the most effective siRNA in inhibiting atrogin-1 expression. As shown in Fig. 4, atrogin-1 was downregulated in the $\mathrm{C} 2 \mathrm{C} 12$ cells transfected with atrogin-1 siRNA-1 and -2 compared to the non-specific siRNA control, yet only siRNA-1 significantly downregulated the expression of atrogin-1 $(\mathrm{P}<0.05)$. Thus, we chose siRNA-1 for subsequent study.

TNF- $\alpha$ induces myotube atrophy and atrogin-1 is overexpressed in the C2C12 cells. After treatment with $10 \mathrm{ng} / \mathrm{ml}$ TNF- $\alpha$, the $\mathrm{C} 2 \mathrm{C} 12$ cells grew slowly and myotubes atrophied with a significantly decreased diameter (Fig. 5A and B). Atrogin-1 was significantly increased at the mRNA and protein levels after treatment with $6 \mathrm{ng} / \mathrm{ml} \mathrm{TNF}-\alpha$ for $2 \mathrm{~h}$ and $10 \mathrm{ng} / \mathrm{ml} \mathrm{TNF}-\alpha$ for $96 \mathrm{~h}$, respectively (Fig. 5C and D).

Atrogin-1 siRNA-1 reduces myotube atrophy induced by TNF- $\alpha$ in the C2C12 cells. To investigate whether inhibition of atrogin-l affects myotube atrophy induced by TNF- $\alpha$, we compared the myotube morphology and myotube diameter in the $\mathrm{C} 2 \mathrm{C} 12$ cells in the atrogin-1 siRNA-1 group and the
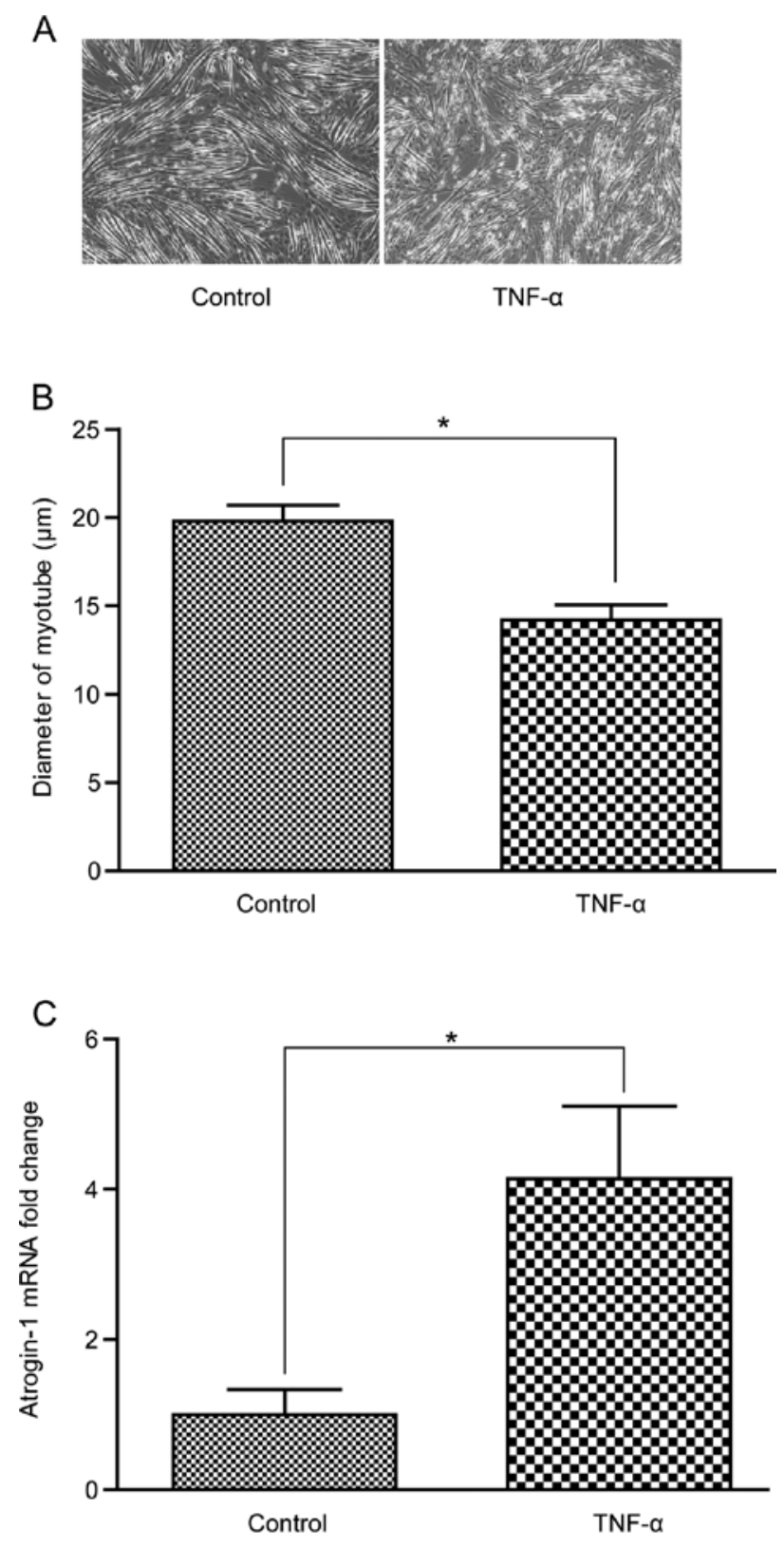

D

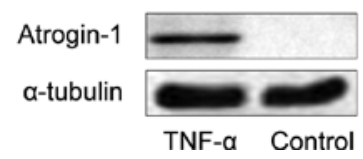

Figure 5. TNF- $\alpha$ induces myotubes atrophy and atrogin-1 overexpression in C2C12 cells. (A) Morphology of the myotubes treated with $10 \mathrm{ng} / \mathrm{ml} \mathrm{TNF-} \alpha$ or without TNF- $\alpha$ (control) showed significant atrophy in the TNF- $\alpha$ group. (B) Myotube diameter in the TNF- $\alpha$ group was significantly decreased compared to the control group. (C) Determination of atrogin-1 mRNA by RT-qPCR showed that atrogin-1 was significantly increased in the TNF- $\alpha$ group compared to the control group. (D) Determination of atrogin-1 protein by western blotting showed the overexpression of atrogin-1 protein in the TNF- $\alpha$ group. *Significant difference $(\mathrm{P}<0.05)$ between the two groups. TNF- $\alpha$, tumor necrosis factor- $\alpha$.

non-specific siRNA control group. No differences were observed in the two groups before incubation with TNF- $\alpha$. However, after treatment with TNF- $\alpha$, the myotubes underwent atrophy with a significant decrease in myotube diameter 


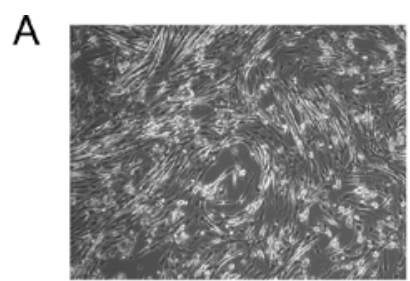

Control

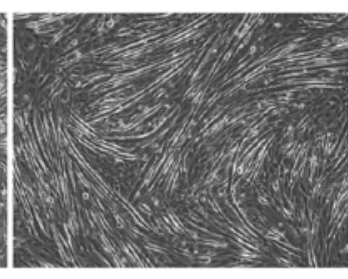

SiRNA-1

B

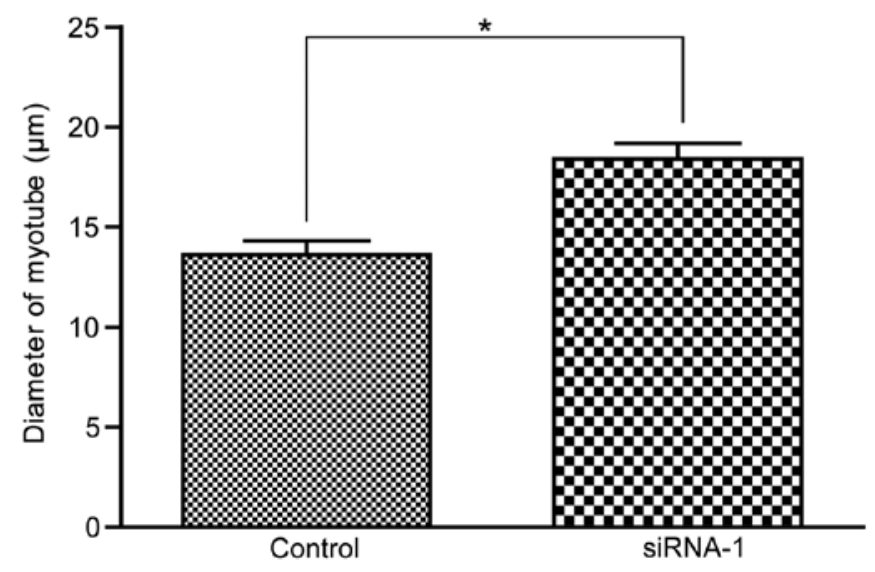

Figure 6. Atrogin-1 siRNA-1 reduces the myotube atrophy induced by TNFin $\mathrm{C} 2 \mathrm{C} 12$ cells. (A) Morphology of the myotubes transfected with atrogin-1 siRNA-1 or non-specific siRNA (control) showed significant atrophy in the control group while no significant atrophy in the atrogin-1 siRNA-1 group (B) Myotube diameter of the atrogin-1 siRNA-1 group increased compared to the control group. *Significant difference $(\mathrm{P}<0.05)$ between the two groups. TNF- $\alpha$, tumor necrosis factor- $\alpha$.

in the control group, while no obvious changes were observed in the atrogin-1 siRNA-1 group (Fig. 6).

\section{Discussion}

In the present study, we demonstrated the involvement of the muscle-specific E3 ubiquitin ligases in muscle atrophy of cancer cachexia in clinical patients, a mouse model and $\mathrm{C} 2 \mathrm{C} 12$ cells. To the best of our knowledge, we firstly report that atrogin-1 and MuRF-1 mRNAs tend to be upregulated in malignant disease patients even before body weight loss. Furthermore, we found that four ubiquitin proteasome pathwayassociated genes (atrogin-1, MuRF-1, ubiquitin and E2-14K) were upregulated in cancer cachexia mice, and knockdown of atrogin-1 protected the $\mathrm{C} 2 \mathrm{C} 12$ cells from atrophy induced by TNF- $\alpha$, implying the potential mechanism underling the muscle wasting of cancer cachexia.

Atrogin-1 and MuRF-1 are elevated in human muscles during various conditions that elicit muscle atrophy. In the present study, atrogin-1 and MuRF-1 mRNA were found to be increased in most of the malignant disease patients, which is consistent with previous research (24). Increased muscle proteasome activity was reported to be correlated with disease severity in gastric cancer patients (21). However, a recent study reported that the expression of atrogin-1 and MuRF-1 in gastric cancer patients was not different from benign disease, and both genes were unaffected by the stage of cancer or the severity of body weight loss, except for a transient increase in mRNA expression of MuRF-1 in patients with stage IV disease relative to those with stage III (25). The inconsistency suggests that atrogin-1 and MuRF-1 expression may be affected by various factors, and studies enrolling more cancer patients are urgently needed to reduce individual differences. In addition, we found that atrogin-1 and MuRF-1 tended to be upregulated even before a visible decline in body weight, suggesting that high expression of atrogin-1 and MuRF-1 may not be an outcome of body weight loss in cancer cachexia patients but may contribute to this process.

Murine adenocarcinoma colon-26-bearing mice exhibit progressive weight loss of skeletal muscle and adipose tissues similar to cancer cachexia; thus, colon-26-induced cachexia in mice has been extensively used to elucidate the mechanisms of cancer cachexia $(26,27)$. In this model, we found that four ubiquitin proteasome pathway-associated genes (atrogin-1, MuRF-1, ubiquitin and E2-14K) were significantly upregulated in the tumor-bearing cancer cachexia mice. These results indicate that the ubiquitin proteasome pathway may be involved in the course of skeletal muscle loss in cancer cachexia. However, whether this pathway plays a key role in this process needs further analysis.

Previous studies indicate that TNF- $\alpha$ plays a critical role in regulating muscle mass and protein degradation during cachexia (28). Atrogin-1 mRNA has been shown to increase with TNF- $\alpha$ exposure in $\mathrm{C} 2 \mathrm{C} 12$ myotubes (29). However, rare studies have reported the effect of siRNA targeting atrogin-1 in resisting the myotube atrophy from the adverse effect of TNF- $\alpha$. In the present study, an effective atrogin-1 siRNA was successfully constructed and an obvious protective effect of atrogin-1 siRNA was observed. These results suggest that knockdown of atrogin-1 may be an effective method to treat muscle atrophy induced by TNF- $\alpha$ in cancer cachexia.

Although our results suggest that muscle-specific ubiquitin ligase E3 may contribute to the muscle atrophy of cancer cachexia, there are several limitations to the present study. Firstly, due to the rare number of cancer cachexia patients, we did not analyze the atrogin-1 and MuRF-1 expression restricted to cancer cachexia patients, but in several types of malignant disease patients. Secondly, due to tissue limitation, the protein levels of either atrogin-1 or MuRF-1 could not be determined in clinical patients. Thirdly, in the mechanistic study, we only focused on the effect of atrogin-1 but not of all associated genes. Studies to investigate the effect and mechanism of other associated genes should be conducted in the future.

In conclusion, the present study demonstrated that atrogin-1 and MuRF-1 are highly expressed in malignant disease patients and cancer cachexia mice. Additionally, knockdown of atrogin-1 protected- myotubes from atrophy induced by TNF- $\alpha$ in C2C12 cells. Given the fact that atrogin-1 and MuRF-1 tend to be upregulated even before visible body weight loss in malignant disease patients, future research may focus on the role of atrogin-1 and MuRF-1 in the early stage of muscle atrophy in cancer cachexia and how to exert the anti-cachectic effects of atrogin-1 and MuRF-1 antagonists. Taken together, our data suggest that targeting atrogin-1 at an early stage of muscle atrophy may be an effective strategy for molecular and clinical intervention in the muscle wasting pathological process in cancer cachexia. 


\section{Acknowledgements}

The present study was supported by the National Natural Science Foundation of China grants (no 30571819).

\section{References}

1. Inui A: Cancer anorexia-cachexia syndrome: Current issues in research and management. CA Cancer J Clin 52: 72-91, 2002.

2. Costelli P and Baccino FM: Cancer cachexia: From experimental models to patient management. Curr Opin Clin Nutr Metab Care 3: 177-181, 2000.

3. Fearon KC, Glass DJ and Guttridge DC: Cancer cachexia: Mediators, signaling, and metabolic pathways. Cell Metab 16: 153-166, 2012

4. Onesti JK and Guttridge DC: Inflammation based regulation of cancer cachexia. Biomed Res Int 2014: 168407, 2014.

5. Ando K, Takahashi F, Motojima S, Nakashima K, Kaneko N, Hoshi K and Takahashi K: Possible role for tocilizumab, an antiinterleukin-6 receptor antibody, in treating cancer cachexia. J Clin Oncol 31: e69-e72, 2013.

6. Gallagher IJ, Stephens NA, MacDonald AJ, Skipworth RJ, Husi H, Greig CA, Ross JA, Timmons JA and Fearon KC: Suppression of skeletal muscle turnover in cancer cachexia: Evidence from the transcriptome in sequential human muscle biopsies. Clin Cancer Res 18: 2817-2827, 2012.

7. Bruera E: ABC of palliative care. Anorexia, cachexia, and nutrition. BMJ 315: 1219-1222, 1997.

8. Nicolini A, Ferrari P, Masoni MC, Fini M,Pagani S, Giampietro O and Carpi A: Malnutrition, anorexia and cachexia in cancer patients: A mini-review on pathogenesis and treatment. Biomed Pharmacother 67: 807-817, 2013.

9. Argilés JM, Costelli P, Carbó N, Pallarés-Trujillo J and LópezSoriano FJ: Tumour growth and nitrogen metabolism in the hos (Review). Int J Oncol 14: 479-486, 1999.

10. Jagoe RT and Goldberg AL: What do we really know about the ubiquitin-proteasome pathway in muscle atrophy? Curr Opin Clin Nutr Metab Care 4: 183-190, 2001.

11. Bonetto A, Aydogdu T, Jin X, Zhang Z, Zhan R, Puzis L, Koniaris LG and Zimmers TA: JAK/STAT3 pathway inhibition blocks skeletal muscle wasting downstream of IL-6 and in experimental cancer cachexia. Am J Physiol Endocrinol Metab 303: E410-E421, 2012.

12. Narsale AA and Carson JA: Role of interleukin-6 in cachexia: Therapeutic implications. Curr Opin Support Palliat Care 8: 321-327, 2014.

13. Bodine SC and Baehr LM: Skeletal muscle atrophy and the E3 ubiquitin ligases MuRF1 and MAFbx/atrogin-1. Am J Physiol Endocrinol Metab 307: E469-E484, 2014.

14. Ogawa T, Furochi H, Mameoka M, et al: Ubiquitin ligase gene expression in healthy volunteers with 20-day bedrest. Muscle Nerve 34: 463-469, 2006.

15. Urso ML, Chen YW, Scrimgeour AG, Lee PC, Lee KF and Clarkson PM: Alterations in mRNA expression and protein products following spinal cord injury in humans. J Physiol 579: 877-892, 2007.
16. Léger B, Vergani L, Sorarù G, Hespel P, Derave W, Gobelet C, D'Ascenzio C, Angelini C and Russell AP: Human skeletal muscle atrophy in amyotrophic lateral sclerosis reveals a reduction in Akt and an increase in atrogin-1. FASEB J 20: 583-585, 2006.

17. Doucet M, Dubé A, Joanisse DR, Debigaré R, Michaud A, Paré MÈ, Vaillancourt R, Fréchette E and Maltais F: Atrophy and hypertrophy signalling of the quadriceps and diaphragm in COPD. Thorax 65: 963-970, 2010.

18. Clavel S, Coldefy AS, Kurkdjian E, Salles J, Margaritis I and Derijard B: Atrophy-related ubiquitin ligases, atrogin-1 and MuRF1 are up-regulated in aged rat Tibialis Anterior muscle. Mech Ageing Dev 127: 794-801, 2006.

19. Dehoux M, Van Beneden R, Pasko N, Lause P, Verniers J, Underwood L, Ketelslegers JM and Thissen JP: Role of the insulin-like growth factor I decline in the induction of atrogin-1/MAFbx during fasting and diabetes. Endocrinology 145: 4806-4812, 2004.

20. Lecker SH, Jagoe RT, Gilbert A, Gomes M, Baracos V, Bailey J, Price SR, Mitch WE and Goldberg AL: Multiple types of skeletal muscle atrophy involve a common program of changes in gene expression. FASEB J 18: 39-51, 2004.

21. Bossola M, Muscaritoli M, Costelli P, Grieco G, Bonelli G, Pacelli F, Rossi Fanelli F, Doglietto GB and Baccino FM: Increased muscle proteasome activity correlates with disease severity in gastric cancer patients. Ann Surg 237: 384-389, 2003.

22. Li YP, Schwartz RJ, Waddell ID, Holloway BR and Reid MB: Skeletal muscle myocytes undergo protein loss and reactive oxygen-mediated NF-kappaB activation in response to tumor necrosis factor alpha. FASEB J 12: 871-880, 1998.

23. Zhou CF, Li XB, Sun H, Zhang B, Han YS, Jiang Y, Zhuang QL, Fang $\mathrm{J}$ and $\mathrm{Wu}$ GH: Pyruvate kinase type M2 is upregulated in colorectal cancer and promotes proliferation and migration of colon cancer cells. IUBMB Life 64: 775-782, 2012.

24. Khal J, Hine AV, Fearon KC, Dejong CH and Tisdale MJ: Increased expression of proteasome subunits in skeletal muscle of cancer patients with weight loss. Int J Biochem Cell Biol 37: 2196-2206, 2005.

25. D'Orlando C, Marzetti E, François S, et al: Gastric cancer does not affect the expression of atrophy-related genes in human skeletal muscle. Muscle Nerve 49: 528-533, 2014.

26. Yasumoto K, Mukaida N, Harada A, et al: Molecular analysis of the cytokine network involved in cachexia in colon 26 adenocarcinoma-bearing mice. Cancer Res 55: 921-927, 1995.

27. Iizuka N, Hazama S, Yoshimura K, Yoshino S, Tangoku A, Miyamoto K, Okita K and Oka M: Anticachectic effects of the natural herb Coptidis rhizoma and berberine on mice bearing colon 26/clone 20 adenocarcinoma. Int J Cancer 99: 286-291, 2002.

28. Pajak B, Orzechowska S, Pijet B, Pijet M, Pogorzelska A, Gajkowska B and Orzechowski A: Crossroads of cytokine signaling - the chase to stop muscle cachexia. J Physiol Pharmacol 59 (Suppl 9): S251-S264, 2008.

29. Li YP, Chen Y, John J, Moylan J, Jin B, Mann DL and Reid MB: TNF-alpha acts via p38 MAPK to stimulate expression of the ubiquitin ligase atrogin1/MAFbx in skeletal muscle. FASEB J 19: 362-370, 2005. 